\title{
Chapter of Gastroenterologists professional guidance on risk mitigation for gastrointestinal endoscopy during COVID-19 pandemic in Singapore
}

Tiing Leong $\underline{A n g^{1,2}}$, FRCPEd, FAMS, James Weiquan $\underline{\underline{L} i^{1,2}}$, MRCP, FAMS, Charles Kien Fong $\underline{V} \underline{u}^{1,3}$, FRACP, FAMS, Gim Hin $\underline{H o}^{1,4}$, MRCP, FAMs, Jason Pik Eu Chang ${ }^{1,5}$, MRCP, FAMs, Chern Hao Chong ${ }^{1,6}$, MRCP, FAMS, Tju Siang $\underline{\text { Chua }}^{1,7}$, FRCPEd, FAMS, David Eng Hui Ong ${ }^{1,8}$, MRCP, FAMs, Benjamin Cherng Hann Yip ${ }^{1,4}$, MRCP, FAMS, Kok Ann $\underline{\mathrm{Gwe}}^{1,9}$, PhD, FAMS

\begin{abstract}
In this paper, we aimed to provide professional guidance to practising gastrointestinal (GI) endoscopists for the safe conduct of GI endoscopy procedures during the current coronavirus disease 2019 (COVID-19) pandemic and future outbreaks of similar severe respiratory tract infections in Singapore. It draws on the lessons learnt during the severe acute respiratory syndrome (SARS) epidemic and available published data concerning the COVID-19 pandemic. It addresses measures before, during and after endoscopy that must be considered for both non-infected and infected patients, and provides recommendations for practical implementation.
\end{abstract}

Keywords: COVID-19, endoscopy, gastroenterology, SARS-CoV2

\section{INTRODUCTION}

This paper aims to provide professional guidance to practising gastrointestinal (Gl) endoscopists for the safe conduct of Gl endoscopy procedures during the coronavirus disease 2019 (COVID-19) pandemic and future outbreaks of similar severe respiratory tract infections in Singapore. It draws on the lessons learnt from the severe acute respiratory syndrome (SARS) epidemic ${ }^{(1)}$ and available published data concerning the COVID-19 pandemic. Considerations that underpin decisionmaking are summarised, and recommended measures to be taken before, during and after Gl endoscopy are provided.

Severe acute respiratory syndrome coronavirus 2 (SARS-CoV-2), which causes the COVID-19 infection, is spread mainly by droplets and close contact, but aerosol-generating procedures (AGPs) as well as environmental and fomite contamination can result in disease transmission. ${ }^{(2,3)}$ SARS-CoV-2 RNA has been detected in stool samples, raising the possibility of faecal-oral transmission. ${ }^{(4,5)}$ As such, both upper and lower Gl endoscopy procedures carry the risk of inadvertent healthcare professional (HCP) exposure and infection, which can result in a nosocomial outbreak with other HCPs and patients being infected. Appropriate processes must be put in place to enhance the safety of both HCPs and patients, utilising limited resources in a rational manner.

To address the issue of safe Gl endoscopy practice during the ongoing COVID-19 pandemic, recommendations have been published by several professional organisations in the West ${ }^{(6-11)}$ and another set of recommendations from China. ${ }^{(12)}$ Although these recommendations have many similarities, there are also subtle differences among them, and some of these recommendations differ from Singapore's current approach towards infection prevention and control. Differences in opinions may arise due to variations in epidemiology and resource availability, and must be contextualised and adapted to best suit the actual needs of the community. ${ }^{(13)}$ Indeed, the sheer amount of information and the speed at which it is disseminated may result in confusion for clinicians serving at the frontlines of healthcare systems that are coming under increasing strain. Hence, the Board of the Chapter of Gastroenterologists, Academy of Medicine, Singapore, decided to publish this concise professional guidance for Gl endoscopists. The practice of evidence-based medicine requires objective scientific data, but where such data is lacking, decisions must be made based on the limited available evidence and, when relevant, extrapolated from indirect data. Endoscopists should keep the safety of both HCPs and patients in mind as the guiding principle while utilising resources in a prudent, cost-effective manner. The key recommendations are summarised in Box 1.

\section{EPIDEMIOLOGICAL CONSIDERATIONS WITH COVID-19 \\ Unique clinical characteristics of SARS-CoV-2 and implications for GI endoscopy}

Performing Gl endoscopy in patients who harbour an increased risk of transmission of communicable diseases is not a new concept. Clear guidelines exist to protect endoscopy staff from communicable diseases during endoscopy, such as the airborne diseases of tuberculosis and varicella. ${ }^{(14,15)}$ SARS-CoV-2, while widely accepted to be transmitted mainly via droplets and contact, theoretically poses a similar threat as airborne diseases

\footnotetext{
${ }^{1}$ Chapter of Gastroenterologists, Academy of Medicine, ${ }^{2}$ Department of Gastroenterology and Hepatology, Changi General Hospital, ${ }^{3}$ Department of Gastroenterology and Hepatology, Tan Tock Seng Hospital, ${ }^{4}$ Division of Gastroenterology, Department of Medicine, Khoo Teck Puat Hospital, ${ }^{5}$ Department of Gastroenterology and Hepatology, Singapore General Hospital, ${ }^{6}$ Gutcare Digestive Liver Endoscopy Associates, ${ }^{7}$ Aliveo Medical, Mount Alvernia Hospital and Mount Elizabeth Hospitals, ${ }^{8}$ Division of Gastroenterology and Hepatology, Department of Medicine, National University Hospital, ${ }^{9}$ Gleneagles Hospital, Singapore

Correspondence: Prof Ang Tiing Leong, Chief and Senior Consultant, Department of Gastroenterology and Hepatology, Changi General Hospital, 2 Simei Street 3, Singapore
} 529889. ang.tiing.leong@singhealth.com.sg 
Box 1. Recommendations for gastrointestinal (GI) endoscopy during coronavirus diseases 2019 (COVID-19) pandemic:

It is recommended that:

1. there is strict adherence to infection prevention and control measures within the endoscopy centre;

2. endoscopists practise meticulous hand hygiene;

3. postponement of non-urgent elective $\mathrm{Gl}$ endoscopy procedures is considered;

4. all patients without a known diagnosis of COVID-19 infection who are being scheduled for elective $\mathrm{Gl}$ endoscopy procedures be risk stratified for possible exposure and likelihood of being an undiagnosed asymptomatic carrier;

5. all patients with recent acute respiratory illness of any severity, with recent travel abroad, on leave of absence/stay home notice, or who had close contact with such individuals should have their elective procedures deferred for at least 14 days to ensure that they do not develop COVID-19 infection;

6. patients with confirmed or suspected COVID-19 infection only undergo emergency $\mathrm{Gl}$ endoscopy procedures that have an immediate impact on management and outcome;

7. when emergency $\mathrm{Gl}$ endoscopy procedures are performed in patients with confirmed or suspected COVID-19 infection, the extent of the procedure and procedure time be limited to only that sufficient for management of the acute problem, deferring more extensive diagnostic sampling or treatment till recovery;

8. enhanced personal protective equipment (PPE) comprising eye protection using goggles/face shield, N95 mask, operating theatre (OT) cap/hairnet, water-resistant gown and gloves be worn for all Gl endoscopy procedures in patients not suspected to have COVID-19 infection during the current pandemic; and

9. in addition to enhanced PPE comprising eye protection using goggles/face shield, N95 mask, OT cap/hairnet, water-resistant gown and gloves, endoscopists consider the use of a powered air-purifying respirator (i.e. PAPR) for $\mathrm{Gl}$ endoscopy procedures in patients with confirmed or suspected COVID-19 infection.

during AGPs. It has been shown that under experimental circumstances, SARS-CoV-2 has similar characteristics to the SARS coronavirus (SARS-CoV) in remaining viable in aerosols for up to three hours. ${ }^{(16)}$ The viability of SARS-CoV-2 on surfaces is variable, depending on the type of surface. It was detectable for up to 72 hours on plastic and stainless steel, while being less viable on copper and cardboard in the same study. ${ }^{(16)}$

Endoscopic procedures should be considered to be AGPs. Scope insertion during upper endoscopy can result in coughing from irritation of the airway and retching from stimulation of the gag reflex. This would generate aerosols that may contain SARSCoV-2 when patients are infected. A study investigating the risk of bacterial exposure to the endoscopist's face during colonoscopy concluded that the endoscopist is exposed to potentially infectious biologic samples while performing colonoscopy. ${ }^{(17)}$ The study used a cut-off of greater than 15 colony-forming units as definite exposure; although it could not prove the clinical significance of such an exposure, it provided a mechanism to highlight how SARS-CoV-2 may potentially be spread during colonoscopy, in which patients may pass flatus with air insufflation. Moreover, studies have shown that live SARS-CoV-2 can be detected in the faeces of infected patients, raising the concern of a faecal- oral route of transmission. ${ }^{(4,5)}$ The well-documented digestive symptoms of COVID-19 also point indirectly to the viability of the virus in the Gl tract. ${ }^{(18)}$ Thus, precautions taken during the performance of colonoscopy should be the same as that for upper endoscopy.

One of the key features of SARS-CoV-2 that may account for its rapid spread, despite increasing knowledge of the disease and the rapid upscaling of responses to curb its spread across and within national borders, is that transmission can occur in asymptomatic individuals. ${ }^{(19,20)}$ Clinical manifestations of the disease range across the entire spectrum of presentations, from an asymptomatic but contagious individual to the patient with acute respiratory distress syndrome. ${ }^{(21-23)}$ This, together with the changing case definitions for suspected cases due to the increasing number of countries facing COVID-19 outbreaks, may blunt the effectiveness of an endoscopy unit's screening protocols to identify suspected and confirmed cases. Even the presence of fever, which is used widely for screening, has come under question as being a hallmark of COVID-19.(21)

Given that a significant number of infected patients may be asymptomatic or have mild disease, contributing to continued community transmission, the COVID-19 pandemic may run a more protracted course. Currently, there is almost universal agreement that all non-urgent Gl endoscopy procedures should be postponed. Comprehensive lists have been published as guidance for what constitutes a prioritised procedure. ${ }^{(8,11)}$ These measures may make sense given the need to reallocate manpower and resources, and the supply chain disruptions affecting the availability of personal protective equipment (PPE). ${ }^{(24)}$ However, it must also be noted that endoscopy provides a minimally invasive modality for diagnostics and therapeutics to pre-empt and prevent the progression of a disease, and can thus help to avoid the need for more invasive or urgent interventions in future. Therefore, when logistically feasible, there is a need to consider measures that would allow us to continue providing such services safely.

\section{CONSIDERATIONS BEFORE GASTROINTESTINAL ENDOSCOPY Organisation of the endoscopy centre Harmonisation of physical structure and functional workflow}

The endoscopy centre is not just a physical construct with space for patient registration, rooms for equipment and procedures, space for disinfection, and recovery beds. There must be harmonisation of the physical design with the optimised daily operational workflow, which is crucial for staff and patient safety, operational efficiency, and ease of implementation of appropriate infection prevention and control measures. Patients should be appropriately sited before and after procedures. There must be a clear demarcation and separation of clean and contaminated zones. The workflow should be such that designated one-way passages are used for the transportation of used or contaminated equipment, separate from clean or disinfected equipment, to avoid cross-contamination. Such a structural design may not be present in older endoscopy centres, which should therefore 
adhere to a clear functional separation for transportation of clean and used equipment to avoid cross-contamination. This concept of physical and functional harmonisation should be taken into account when planning new endoscopy units. This is crucial during the current pandemic but remains very important during normal times.

\section{Patient flow for endoscopy procedures}

The flow of patients should utilise the existing endoscopy infrastructure to derive appropriate infection prevention and control measures for the safety of the patients and staff. In Singapore, triage occurs before patients and relatives enter the hospital premises. All outpatients registering within the physical boundaries of the endoscopy facility would have been adequately screened prior to entering the endoscopy reception area. All frontline staff performing such screening or in contact with patients are required to wear a surgical face mask. A second layer of screening should occur within the endoscopy centre. The principle of physical distancing should be adhered to in all aspects of the patient journey, from queuing in the reception to the waiting area before endoscopy. Inpatients would have undergone the requisite screening on admission and, in some cases, may even have been tested for and confirmed to be negative for COVID-19 prior to endoscopy.

There should not be outpatients with confirmed or suspected COVID-19 infection undergoing elective endoscopy. Only emergency endoscopy procedures should be performed for such patients, in which case they should already have been hospitalised. The procedures should ideally be performed in dedicated negative pressure rooms located in the endoscopy centre, high dependency or intensive care units, and operating theatres (OTs). While newer facilities would certainly have built such dedicated negative pressure rooms in the endoscopy centre and OTs, these may be lacking in older facilities, and to mitigate risk, procedures must be performed with portable HEPA (high-efficiency particulate absorbing) filter units placed in the room and set to maximum output, behind closed doors. These older facilities should also plan for such dedicated negative pressure rooms in future. COVID-19 patients would have varying degrees of respiratory compromise, ranging from not requiring oxygen supplementation to needing intubation and mechanical ventilation. If a transfer from the isolation room to the endoscopy centre is required for the procedure to be performed, such patients should wear a face mask and be transferred directly from the ward isolation room to the endoscopy room, and vice versa after the procedure is completed. This minimises waiting time and the risk of cross-infection of other patients. The transfer route should be planned in advance. Additionally, the lift used to transfer the patient should be dedicated to the patient transfer, and no other individuals should remain within the same lift while the transfer is in process. Intubated patients who require emergency endoscopy in the OT should likewise be directly transferred to the dedicated OT and then sent back to the intensive care unit directly after completion of the procedure, without transit in any holding areas. Staff attending to these
COVID-19-positive patients must wear PPE comprising an N95 mask, a water-resistant gown and gloves.

\section{Keeping track of personal protective equipment inventory} Ensuring an adequate supply of PPE is essential for the protection of HCPs. The fragility of the global supply chain for PPE necessitates greater involvement of the endoscopy centre's director and manager in PPE stocktaking and in maintaining an overview of its rate of usage in relation to projected resupply. It is recommended that the appropriate personnel work with their counterparts in Ambulatory Surgery and OTs, as a decline in elective procedures from other departments may mean that a ready supply of unutilised PPE may be available to counter surges in demand in the endoscopy centre.

\section{Indications for endoscopy and screening of patients Patient selection for endoscopy}

The indication and contraindications for endoscopy remain the same regardless of the existence of a pandemic. The main issue is resource availability in times of crisis and how to optimise the use of limited resources for the greater good. The principles of patient selection in the COVID-19 pandemic can be better appreciated if one understands the macro environment in which the endoscopy unit functions. Postponing non-urgent elective cases and prioritising cases listed for endoscopy may free up manpower for other medical exigencies; minimise patient movement to decrease the risk of infection transmission to other patients and HCPs; and, in certain cases, make available additional beds and physical space to battle the COVID-19 situation.

When a country is completely overwhelmed by an outbreak, all elective procedures would cease and the limited resources left for Gl endoscopy would be channelled towards only life-saving emergency therapeutic Gl endoscopy procedures. When the outbreak is still contained and if resources permit, it is possible to continue performing routine $\mathrm{Gl}$ endoscopy procedures that cannot be deferred for too long for patients without COVID-19 infection. It must be emphasised that while an approach of deferring all non-urgent procedures during the pandemic may receive broad consensus, a postponed non-urgent procedure may, over the course of time, require emergency intervention due to disease progression. This is particularly relevant if the pandemic fails to be controlled and lasts for a longer period of time. Examples of these scenarios would be variceal surveillance and a patient with a positive faecal immunohistochemistry test.

Patients who are diagnosed or suspected to have COVID-19 infection should not undergo non-urgent GI endoscopy procedures. These procedures should be deferred until full recovery in order to ensure both staff and patient safety. Only emergency $\mathrm{Gl}$ endoscopic procedures that have an immediate impact on patient management should be performed.

\section{Risk stratification for likelihood of harbouring occult COVID-19 infection}

It is important to perform patient screening and risk stratification before listing a patient for an elective procedure. The aim is to 
identify patients who may potentially be infected or are at risk of being infected and to reschedule their elective endoscopy to a later date, when it is clearer that they are not infected.

Risk stratification is mainly based on screening questions focusing on patient epidemiological and clinical risk factors, such as fever, respiratory symptoms, travel, occupation, contact history and clustering. Guidelines from the West on scheduling of patients without a diagnosis of COVID-19 infection for GI endoscopy considered factors such as presence of acute respiratory symptoms, exposure to contacts with such symptoms, and recent travel to high-risk countries as being important for risk stratification. They recommended the arrangement of separate pre- and post-endoscopy recovery areas for such high-risk cases undergoing endoscopy. Patients were stratified as either low or high risk in a paper by the European Society of Gastrointestinal Endoscopy ${ }^{(11)}$ and as low, moderate or high risk in a paper from Italy. ${ }^{(7)}$ Singapore has local transmission clusters and all travellers returning from abroad are regarded as being at high risk to be infected during travel. Hence, it is prudent to defer non-emergency $\mathrm{Gl}$ endoscopy procedures by at least 14 days, the incubation period of the infection, for all patients with acute respiratory symptoms of any severity, who recently returned from overseas, or who are on isolation with a leave of absence or stay home notice, as well as those who had contact with such individuals. The rationale for deferment of at least 14 days is to avoid having patients who are contagious from spreading the disease while in an asymptomatic stage. Elective endoscopy should only be performed for low-risk patients.

\section{CONSIDERATIONS DURING ENDOSCOPY Performing gastrointestinal endoscopy procedures}

If resources permit, routine diagnostic and therapeutic GI endoscopy procedures can still be performed as usual and in a timely manner in the endoscopy centre for COVID-19-negative patients during the disease outbreak.

For patients with confirmed or suspected COVID-19 infection, only emergency $\mathrm{Gl}$ endoscopic procedures that have an immediate impact on patient management should be performed, such as endoscopic haemostasis for GI bleeding, or endoscopic retrograde cholangiopancreatography for biliary tract decompression in severe cholangitis. When emergency $\mathrm{Gl}$ endoscopy is performed for infected patients, the extent of the procedure and procedure time should be limited to that sufficient for treatment of the acute problem, with deferment of more extensive diagnostic sampling or treatment till recovery in order to reduce the risk of HCP exposure. One example is to secure haemostasis for ulcer bleeding without additional biopsies for histology or rapid urease test, while another is insertion of a plastic stent to decompress a biliary system that was obstructed due to a large stone, and to defer cholangioscopy with laser lithotripsy to a later date after recovery.

\section{Types of personal protective equipment worn during gastrointestinal endoscopy}

For COVID-19-negative patients, it is recommended that an enhanced PPE be used for all Gl endoscopy procedures during the current COVID-19 pandemic and future outbreaks of similarly severe highly contagious respiratory tract infections. This enhanced PPE consists of an N95 face mask, eye protection with goggles or a face shield, an OT cap or hairnet, a water-resistant gown, and gloves. Hand hygiene must be practised when PPE is worn and removed. All endoscopy staff must be fitted for masks and adequately trained in the use of PPE. During an elective list, it may be possible to just change gloves in between cases for patients who do not have COVID-19 infection. If the PPE is soiled, or when performing endoscopy in patients with known transmissible diseases such as methicillin-resistant Staphylococcus aureus or vancomycin-resistant Enterococcus, the gown must be changed. The actual magnitude of the risk of GI endoscopy procedures causing COVID-19 transmission is unclear. The British Society of Gastroenterology has recommended enhanced PPE only for upper $\mathrm{Gl}$ endoscopy procedures and the water-repellent surgical face mask and full arm-length disposable apron for lower Gl procedures, with consideration of the use of enhanced PPE in high-risk or COVID-19-positive patients during lower Gl endoscopy. ${ }^{(6)}$ A paper from Italy suggested that for low-risk patients undergoing lower Gl endoscopy, a surgical face mask may suffice. ${ }^{(7)}$ These recommendations were opinions made with a view of conserving PPE. As procedures could conceivably be performed in asymptomatic undiagnosed patients due to disease spread in the community and an endoscopy list generally consists of a mix of both upper and lower endoscopy cases, the N95 mask is recommended in Singapore for all Gl endoscopy procedures. In China, PPE at Biosafety Level 3 is recommended for all Gl endoscopy procedures during the pandemic. ${ }^{(12)}$

For confirmed or suspected cases of COVID-19 infection needing emergency $\mathrm{Gl}$ endoscopy procedures, the use of PAPRs in addition to the enhanced PPE, if they are available, provides an additional safety barrier and should be strongly considered. It is recognised that wearing PPE can potentially affect the performance of the endoscopist and that a lack of familiarity with the PAPR device, such that it is not worn or disinfected properly, may potentially place the endoscopist at risk of infection. As mentioned, while guidelines in the West only recommended the use of N95 masks in such situations, PPE at Biosafety Level 3 is required in China during all Gl endoscopy procedures. ${ }^{(12)}$ Although a case report of HCPs exposed to AGPs for a COVID-19 patient suggested that surgical masks, hand hygiene and other standard procedures may possibly be adequate protection, it could simply be fortuitous. ${ }^{(25)}$ Given the potentially devastating consequences, caution is advised and the use of full PPE is recommended.

\section{REQUIREMENTS AFTER ENDOSCOPY Post-procedure patient monitoring}

After endoscopy, standard post-monitoring care should be instituted. For COVID-19-positive patients who are sedated for endoscopy, one should consider the use of reversal agents to enhance the recovery process.

\section{Importance of disinfection}

Care must be taken to avoid cross-contamination of disinfected equipment by contaminated endoscopes during the process of 
transportation for disinfection after a procedure. Endoscopes and accessories must be reprocessed according to existing guidelines. ${ }^{(26)}$ Environmental decontamination within the room is essential to reduce the risk of fomite transmission after each procedure, especially for surfaces frequently in contact with patients and endoscopists. At the end of each endoscopy list, or if there is gross contamination after a procedure, an in-depth cleaning process followed by disinfection is required. If the room has been used for a COVID-19-infected patient, terminal cleaning of the room must be performed using full PPE, in accordance with the hospital environmental services standard terminal cleaning procedure. Although most guidelines on the COVID-19 outbreak have emphasised PPE measures, the single most effective protection against transmission of infections by endoscopy is proper reprocessing and disinfection.

\section{CONCLUSION}

Effective infection control and prevention measures are essential to prevent iatrogenic disease transmission. A clear workflow must be established for both screening and risk stratification and for conducting procedures, with appropriate use of PPE. These safeguards are needed to protect both HCPs and patients.

\section{REFERENCES}

1. Shaw K. The 2003 SARS outbreak and its impact on infection control practices. Public Health 2006; 120:8-14.

2. Wu F, Zhao S, Yu B, et al. A new coronavirus associated with human respiratory disease in China. Nature 2020; 579:265-9.

3. Wu YC, Chen CS, Chan YJ. The outbreak of COVID-19: an overview. J Chin Med Assoc 2020; 83:217-20.

4. Wang W, Xu Y, Gao R, et al. Detection of SARS-CoV-2 in different types of clinical specimens. JAMA 2020; 323:1843-4.

5. Xiao F, Tang M, Zheng X, et al. Evidence for gastrointestinal infection of SARSCoV-2. Gastroenterology 2020; 158:1831-3.

6. British Society of Gastroenterology. BSG/JAG COVID-19 guidance and advice. Available at: https://www.bsg.org.uk/covid-19-advice. Accessed March 27, 2020.

7. Repici A, Maselli R, Colombo M, et al. Coronavirus (COVID-19) outbreak: what the department of endoscopy should know. Gastrointest Endosc 2020; 92:192-7.

8. American Society for Gastrointestinal Endoscopy. Joint Gl society message: COVID-19 clinical insights for our community of gastroenterologists and gastroenterology care providers. Available at: https://www.asge.org/home/jointgi-society-message-covid-19. Accessed March 27, 2020.
9. Gastroenterological Society of Australia. Considerations for Australian endoscopy units during the COVID-19 pandemic. Available at: https://www. gesa.org.au/resources/covid-19. Accessed March 27, 2020.

10. Tse F, Borgaonkar M, Leontiadis GI. COVID-19: advice from the Canadian Association of Gastroenterology for endoscopy facilities, as of March 16, 2020. In: Canadian Association of Gastroenterology [online]. Available at: https:// www.cag-acg.org/images/publications/CAG-Statement-COVID-\&-Endoscopy. pdf. Accessed March 27, 2020.

11. European Society of Gastrointestinal Endoscopy. ESGE and ESGENA Position Statement on gastrointestinal endoscopy and the COVID-19 pandemic. Available at: https://www.esge.com/esge-and-esgena-position-statement-ongastrointestinal-endoscopy-and-the-covid-19-pandemic/. Accessed March 27, 2020

12. Zhang Y, Zhang X, Liu L, Wang H, Zhao Q. Suggestions for infection prevention and control in digestive endoscopy during current 2019-nCoV pneumonia outbreak in Wuhan, Hubei province, China. Endoscopy 2020; 52:312-4.

13. Ang TL. Gastrointestinal endoscopy during COVID-19 pandemic. J Gastroenterol Hepatol 2020; 35:701-2.

14. ASGE Quality Assurance in Endoscopy Committee, Calderwood AH, Day LW, et al. ASGE guideline for infection control during GI endoscopy. Gastrointest Endosc 2018; 87:1167-79.

15. Sehulster L, Chinn RY; CDC; HICPAC. Guidelines for environmental infection control in health-care facilities. Recommendations of CDC and the Healthcare Infection Control Practices Advisory Committee (HICPAC). MMWR Recomm Rep 2003; 52(RR-10):1-42.

16. van Doremalen N, Bushmaker T, Morris DH, et al. Aerosol and surface stability of SARS-CoV-2 as compared with SARS-CoV-1. N Engl J Med 2020; 382:1564-7.

17. Johnston ER, Habib-Bein N, Dueker JM, et al. Risk of bacterial exposure to the endoscopist's face during endoscopy. Gastrointest Endosc 2019; 89:818-24.

18. Wong SH, Lui RN, Sung JJ. Covid-19 and the digestive system. J Gastroenterol Hepatol 2020; 35:744-8.

19. Tong ZD, Tang A, Li KF, et al. Potential presymptomatic transmission of SARSCoV-2, Zhejiang Province, China, 2020. Emerg Infect Dis 2020; 26:1052-4.

20. Rothe C, Schunk M, Sothmann P, et al. Transmission of 2019-nCoV infection from an asymptomatic contact in Germany. N Engl J Med 2020; 382:970-1.

21. Guan WJ, Ni ZY, Hu Y, et al. Clinical characteristics of coronavirus disease 2019 in China. N Engl J Med 2020; 382:1708-20.

22. Huang C, Wang Y, Li X, et al. Clinical features of patients infected with 2019 novel coronavirus in Wuhan, China. Lancet 2020; 395:497-506.

23. Chen N, Zhou M, Dong X, et al. Epidemiological and clinical characteristics of 99 cases of 2019 novel coronavirus pneumonia in Wuhan, China: a descriptive study. Lancet 2020; 395:507-13.

24. World Health Organization. Rational use of personal protective equipment for coronavirus disease 2019 (COVID-19). Available at: https://apps.who.int/iris/ bitstream/handle/10665/331215/WHO-2019-nCov-IPCPPE_use-2020.1-eng. pdf. Accessed March 27, 2020.

25. Ng K, Poon BH, Kiat Puar TH, et al. COVID-19 and the risk to health care workers: a case report. Ann Intern Med 2020; 172:766-7.

26. Beilenhoff $U$, Biering $H$, Blum R, et al. Reprocessing of flexible endoscopes and endoscopic accessories used in gastrointestinal endoscopy: Position Statement of the European Society of Gastrointestinal Endoscopy (ESGE) and European Society of Gastroenterology Nurses and Associates (ESGENA) - Update 2018. Endoscopy 2018; 50:1205-34. 